\title{
Effect of Antiarrhythmic Drug Therapy on the Incidence of Shocks in Patients who Receive an Implantable Cardioverter Defibrillator after a Single Episode of Sustained Ventricular Tachycardia/Fibrillation
}

\author{
WILLIAM H. KOU, * MARVIN M. KIRSH,† STEVE F. BOLLING, $†$ \\ MACK STIRLING, ${ }^{*}$ ALAN H. KADISH, ${ }^{*}$ MICHAEL DE BUITLEIR, ${ }^{* *}$ \\ HUGH CALKINS, ${ }^{*}$ * RUTH R.R. LEWIS, ${ }^{* *}$ and FRED MORADY** \\ From the *Ann Arbor Veterans Administration Medical Center; and the **Division of \\ Cardiology, Department of Internal Medicine and the tDivision of Thoracic Surgery, Department \\ of Surgery, University of Michigan Hospital, Ann Arbor, Michigan
}

\begin{abstract}
KOU, W. H., ET AL.: Effect of Antiarrhythmic Drug Therapy on the Incidence of Shocks in Patients who Receive an Implantable Cardioverter Defibrillator after a Single Episode of Sustained Ventricular Tachycardia/Fibrillation. Seventy-four patients ( 16 women, 58 men, age $58 \pm 11$ years, mean \pm standard deviation) who received an implantable cardioverter defibrillator (ICD) after experiencing a single episode of ventricular tachycardia or ventricular fibrillation were followed to determine if antiarrhythmic drug therapy affects the incidence of ICD discharges. Thirty-three patients (group A) were treated with an antiarrhythmic drug that was either untested or previously demonstrated during electropharmacological testing to be ineffective in suppressing the induction of ventricular tachycardia. Forty-one patients (group B) were not treated with an antiarrhythmic drug. There were no significant differences between the two groups in regards to age, sex, incidence of coronary artery disease, left ventricular function or the type of ICD pulse generator used. During a mean follow-up of 14 months for the entire cohort, 15 patients $(46 \%)$ in group A and 18 patients (44\%) in group B experienced at least one ICD shock. The time to the first appropriate shock ( $5 \pm 5$ months in both groups) and the frequency of ICD shocks $(0.3 \pm 0.2 /$ month in group A vs $0.4 \pm 0.5 /$ month in group B) were similar in both groups. The incidence of syncope at the time of ICD discharge was higher in group $A$ than group $B$ patients $(31 \%$ vs $5 \%, P<0.05)$. In conclusion, antiarrhythmic drugs that are untested or have failed electropharmacological testing do not appear to reduce the probability of ICD discharge over a short-term (mean 14 months) follow-up in patients who have had only one clinical episode of VT/VF and may increase the risk of syncope during ICD discharge. Studies with a larger sample size and longer follow-up period will be needed to confirm these findings. (PACE, Vol. 14, November, Part I 1991)
\end{abstract}

implantable cardioverter defibrillator

\section{Introduction}

Patients who have life-threatening ventricular tachyarrhythmias and who do not respond to

Address for reprints: William H. Kou, M.D., Division of Cardiology (111A), Ann Arbor Veterans Affairs Medical Center, 2215 Fuller Road, Ann Arbor, MI 48105. Fax: (313) 761-5398.

Received December 6, 1990; revision April 8, 1991; revision July 23, 1991; accepted August 13, 1991. pharmacological therapy may undergo implantation of an implantable cardioverter defibrillator (ICD). Although the ICD may be lifesaving, the shocks that are delivered are painful. ${ }^{1-3}$ It has been common clinical practice to prescribe antiarrhythmic drug therapy for some of these patients, in hopes of minimizing the number of ICD shocks. ${ }^{4,5}$ However, it is unclear whether this practice actually reduces the incidence of ICD shocks. Therefore, the purpose of this study was to determine the effect of antiarrhythmic drug 
therapy on the incidence of ICD shocks. In order to avoid a possible bias towards treating patients who had frequent episodes of ventricular tachycardia (VT) or ventricular fibrillation (VF), this study was limited to patients who had only one episode of VT or VF before undergoing implantation of the ICD.

\section{Methods}

\section{Patient Population}

Between October, 1986 and September, 1989, 104 patients underwent implantation of an ICD at the University of Michigan Hospital and the Ann Arbor Veterans Affairs Medical Center. During the immediate postoperative period, six patients died and one patient had the ICD system removed because of infection. During follow-up, seven patients received at least one ICD shock that was documented to have been inappropriate. These 14 patients were excluded from the study. In order to avoid bias towards treating patients with frequent arrhythmias, also excluded were 16 patients who had a history of recurrent VT or VF before undergoing ICD implantation. The study group consisted of the remaining 74 patients who were referred after a single episode of ventricular tachyarrhythmias; five patients presented with sustained VT that had caused near syncope, whereas 69 patients had sustained VT or VF that resulted in syncope or cardiac arrest necessitating direct current countershock during resuscitation. There were 14 women and 60 men with a mean age of 59 \pm 12 years ( \pm standard deviation). Among these 74 patients, 55 had coronary artery disease, nine had an idiopathic dilated cardiomyopathy, two had valvular heart disease, one had a hypertrophic cardiomyopathy, and seven had no identifiable structural heart disease.

Each patient underwent a baseline electrophysiological test. The stimulation protocol included pacing at two right ventricular sites, using two basic drive train cycle lengths (600 or 500 $\mathrm{msec}$, and $400 \mathrm{msec}$ ) and up to three extrastimuli. If sustained monomorphic VT was not induced, intravenous administration of isoproterenol (2 mg/ $\min$ ) was used to facilitate induction of VT. If sustained monomorphic VT was induced, electropharmacological testing was performed. If effec- tive pharmacological therapy could not be identified and if the patient was not an appropriate candidate for endocardial resection or catheter ablation, an ICD was implanted. An ICD was also implanted if sustained monomorphic VT was not inducible and a reversible cause for the VT/VF could not be identified.

\section{Implantation of the ICD System}

All of the ICD leads and pulse generators were manufactured by Cardiac Pacemakers, Inc. (St. Paul, MN, USA). Eight patients received a transvenous rate sensing lead at the right ventricular apex, a transvenous superior vena cava spring-coil defibrillation electrode at the mid-right atrium, and an epicardial defibrillation patch sewn on the left ventricle through a left thoracotomy. In the other patients, a median sternotomy approach was used to place a pair of screw-in, epicardial rate sensing leads on the anterior surface of the right ventricle and a pair of defibrillation patch electrodes on the lateral left ventricle and on the right ventricle. The energy required to terminate VT and/or VF was determined and in each patient was lower than the delivered energy of the pulse generator (25-31 joules).

Concomitant coronary artery bypass grafting surgery was performed on 13 patients of whom five were later discharged on an antiarrhythmic regimen. There was another patient who had undergone percutaneous transluminal coronary angioplasty 2 days before the implantation of ICD. This patient was later discharged on no antiarrhythmic regimen.

At the discretion of the attending or referring physicians, 33 patients (group A) were treated long-term with an antiarrhythmic drug (Table I). In 16 of these 33 patients, the antiarrhythmic drug had been demonstrated to be ineffective in suppressing the induction of VT during electropharmacological testing. In 17 patients, the efficacy of the antiarrhythmic drug had not been tested.

The remaining 41 patients (group B) were not treated with an antiarrhythmic drug after implantation of the ICD.

\section{Follow-Up}

Patients were seen in an outpatient clinic every 2 months during the first year after the im- 
Table I.

Antiarrhythmic Drugs Used in Group A Patients

\section{Number of Patients}

$\begin{array}{lr}\text { Amiodarone } & 15 \\ \text { Quinidine } & 5 \\ \text { Beta Blocker } & 5 \\ \text { Encainide } & 3 \\ \text { Mexiletine } & 2 \\ \text { Procainamide } & 1 \\ \text { Quinidine + Beta Blocker } & 1 \\ \text { Amiodarone + Mexiletine } & 1\end{array}$

plantation and every month thereafter. In patients who experienced ICD shocks, the activity at the time of shock and any associated symptoms were recorded. The ICD discharges were confirmed by the patient pulse count measured by the AIDCHECK (Cardiac Pacemakers, Inc.) during each visit. The shock was considered appropriate if it was associated with premonitory symptoms or if it occurred during sleep. Shocks that occurred during exercise were also considered appropriate only if the maximum sinus rate during a treadmill exercise test was lower than the rate cutoff of the ICD. Ambulatory electrocardiographic or inpatient telemetric monitoring was performed in patients who had experienced frequent ICD discharges in an attempt to document the arrhythmias that triggered the discharges.

\section{Data Analysis}

All values are expressed as mean \pm standard deviation. Between group comparison were performed by Chi-square analysis or Student's t-test. Kaplan-Meier survival curve analysis was used to display the difference of ICD shocks between groups. The significance of difference was determined by Wilcoxon test. A P value $<0.05$ was considered significant for all statistical tests.

\section{Results}

\section{History of Ventricular Tachyarrhythmias and Results of Electrophysiological Study}

In group A, 31 patients had suffered a cardiac arrest due to VT or VF, and two patients presented with presyncope during which sustained monomorphic VT was documented. A baseline electrophysiological test demonstrated inducible sustained VT in 23 patients (monomorphic in 18 and polymorphic in five). The VT was induced by a single extrastimulus in five, double extrastimuli in seven and triple extrastimuli in 11 patients. The mean cycle length of the induced VT was $268 \pm$ $63 \mathrm{msec}$.

In Group B, 35 patients had suffered a cardiac arrest due to VT or VF, four patients presented with presyncope during which sustained monomorphic VT was documented, and two patients presented with unexplained syncope, both with a history of anterior wall myocardial infarction. A baseline electrophysiological test demonstrated inducible sustained VT in 30 patients (monomorphic in 24 and polymorphic in six). The VT was induced by a single extrastimulus in four, double extrastimuli in 13, and triple extrastimuli in 13 patients. The mean cycle length of the induced VT was $252 \pm 69 \mathrm{msec}$. There was not a significant difference in the mean cycle length of the induced VT between the two groups.

\section{Incidence of ICD Shock}

During a mean follow-up period of $14 \pm 8$ months (range 4-33 months), three patients had the device removed after undergoing a heart transplantation surgery and four patients died. One of the deaths was sudden. These patients were censored at the time of device removal or death. Overall, $33 / 74$ patients $(45 \%)$ experienced $\geq 1$ ICD shocks that were presumably appropriate, i.e., no shocks that occurred during a rhythm other than VT or VF was documented. The first of these shocks occurred at mean of $5 \pm 6$ months postimplantation. Thirteen patients experienced the first shock within the first 2 months of follow-up, 24 patients by the sixth month, and 29 by the end of the first year. Premonitory symptoms, including sudden onset of palpitation, dizziness, shortness of breath, presyncope or syncope preceding each ICD shock occurred in 13 patients, preceding some of the shocks in ten patients, and never occurred in eight patients. There were two other patients who experienced ICD shocks only during sleep.

\section{Antiarrhythmic Therapy and ICD Shocks}

The ICD shocks occurred in 15/33 patients $(46 \%)$ who were being treated with antiarrhythmic 
Table II.

Patient Characteristics

\begin{tabular}{lcc}
\hline & Group A & Group B \\
\hline Number of Patients & 33 & 41 \\
Males/Females & $25 / 8$ & $33 / 8$ \\
Age (years) & $60 \pm 10$ & $56 \pm 13$ \\
Coronary Artery Disease & 25 & 31 \\
Left Ventricular Ejection Fraction & $0.32 \pm 0.15$ & $0.33 \pm 0.15$ \\
Concomitant Coronary Revascularization Operation & 5 & 9 \\
Clinical Presentation & 2 & 3 \\
$\quad$ Presyncope & 31 & 38 \\
$\quad$ Syncope or Cardiac Arrest & & 31 \\
$\quad$ Sensing Criterion of ICD & 27 & 10 \\
$\quad$ Rate & 6 & $11 \pm 7^{*}$ \\
Duration + PDF & $17 \pm 8$ & \\
\hline
\end{tabular}

${ }^{\star} \mathrm{P}<0.05 ; \mathrm{ICD}=$ implantable cardioverter defibrillator; $\mathrm{PDF}=$ probability density function; $\mathrm{VT}=$ ventricular tachycardia.

drugs (group A) and 18/41 patients (44\%) who were not receiving any arrhythmic therapy (group B) $(P>0.05)$. If those seven patients (three were taking antiarrhythmic drugs and four were not) who had experienced inappropriate ICD shocks were included, the incidence of ICD shocks was $18 / 36(50 \%)$ in group A and $22 / 45(49 \%)$ in group $\mathrm{B}(\mathrm{P}>0.05)$. There were also no significant differences between these two groups of patients in re- gards to mean age, sex, incidence of coronary artery disease, left ventricular function, indications of ICD implantation, and type of ICD pulse generators used (Table II). However, the follow-up period was longer in group A ( $17 \pm 8$ months vs $12 \pm 8$ months, $\mathrm{P}<0.05$ ). Kaplan-Meier survival curve analysis for patients who remained free of presumably appropriate ICD shocks are shown in Figure 1 and for patients who remained free of any ICD

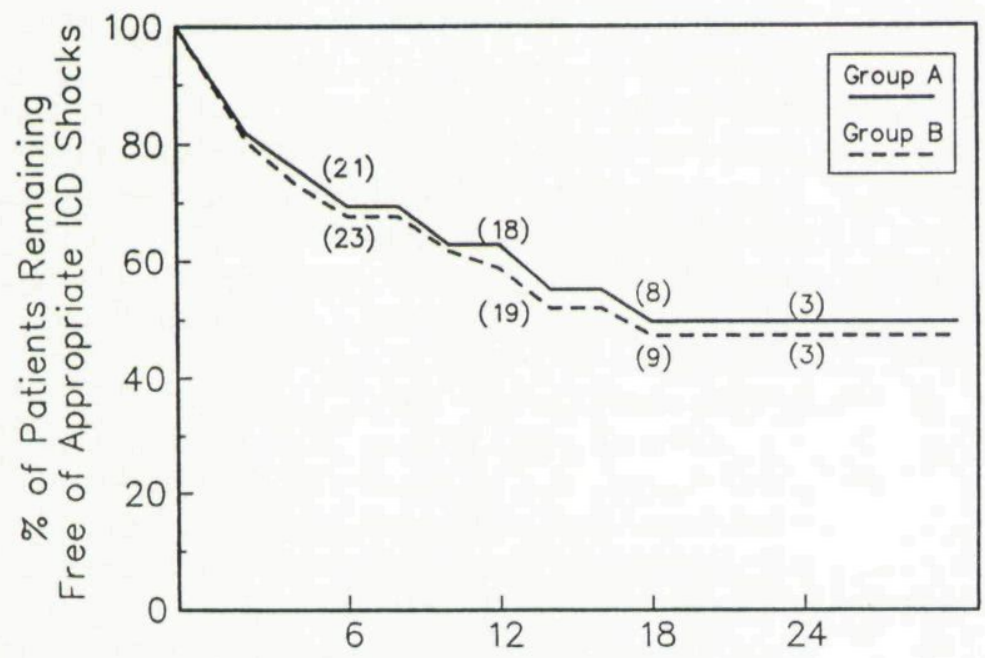

Month After ICD Implantation
Figure 1. Kaplan-Meier curves for patients remaining free of appropriate implantable cardioverter defibrillator shock during follow-up are similar in both groups. Number in parenthesis indicates number of patients remained free of ICD shocks. 
KOU, ET AL.

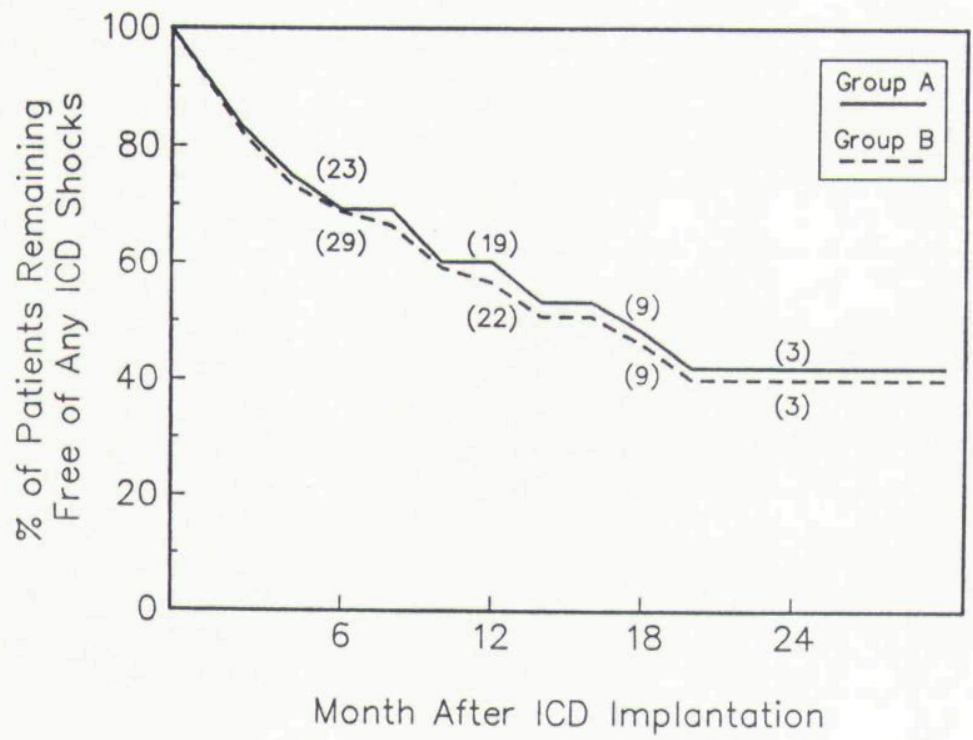

Figure 2. Kaplan-Meier curves for patients remaining free of any implantable cardioverter defibrillator shock during follow-up are also similar in both groups. Number in parenthesis indicates number of patients remaining free of ICD shocks.

shocks are shown in Figure 2. In either case, the curves indicated no difference in patients remaining free of ICD shocks between these two groups of patients. The percentage of freedom of ICD shocks in patients in whom no inappropriate shock was documented at $6,12,18$, and 24 months were $69 \%, 63 \%, 50 \%$, and $50 \%$ for group A; and $68 \%, 59 \%, 47 \%$, and $47 \%$ for group B patients (P $>0.05$, power 0.45 ), and that of all ICD shocks were $69 \%, 60 \%, 48 \%$, and $42 \%$ for group A; and $69 \%, 57 \%, 46 \%$, and $40 \%$ for group B patients (P $>0.05$, power 0.53 ).

\section{Untested Versus Ineffective Antiarrhythmic Drugs in Group A Patients}

In group A, 17 patients were treated with an untested antiarrhythmic drug and 16 were treated with an antiarrhythmic drug that failed electropharmacological testing. Seven patients (41\%) treated with an untested drug and eight patients $(50 \%)$ treated with an ineffective drug experienced ICD shocks during follow-up $(\mathrm{P}>0.05)$.

\section{Time to the First Shock and Frequency of Shocks}

The first ICD shock occurred at a mean postoperative interval of $5 \pm 5$ months in group A and group B. The frequency of ICD shocks in group A was $0.3 \pm 0.2$ shocks/month, compared to $0.4 \pm$ 0.5 shocks/month in group B ( $\mathrm{P}>0.05)$.

\section{Level of Consciousness at Time of ICD Shocks}

Among the 13 patients in group A who had experienced ICD shocks and also had a history of loss of consciousness in association with spontaneous VT or VF, four of these patients (31\%) also experienced loss of consciousness at the time of ICD discharge, including the patient who died suddenly. In contrast, among the 18 patients in group B who had experienced ICD shocks and also had a history of loss of consciousness during spontaneous VT or VF, only one (5\%) experienced syncope during the ICD discharge $(\mathrm{P}<0.05)$. Among these five patients who experienced loss of consciousness at time of ICD shocks, only one is on a beta blocker medicine. While among the remaining 26 patients who did not experience loss of consciousness during ICD shocks, nine were also taking either a beta blocker or a calcium channel blocker medicine $(\mathrm{P}>0.05)$.

There were five patients, two in group A and three in group B, who presented without a history of syncope. Both patients in group A experienced ICD shocks and none had loss of consciousness. Only one of the three patients in group B experienced ICD shock and that patient did not pass out at the time of the shock. 


\section{Discussion}

In this study, patients with an ICD provided an opportunity to examine whether antiarrhythmic drugs that had not been demonstrated to suppress the induction of VT might decrease the frequency of VT/VF in a population at high risk of lethal ventricular tachyarrhythmias. In patients who had only one episode of sustained VT or VF before receiving an ICD, treatment with antiarrhythmic agents that were either untested or ineffective in suppressing the induction of VT did not influence the incidence of ICD shocks. The antiarrhythmic drug therapy neither prolonged the time to the first shock nor reduced the frequency of ICD shocks during short-term follow-up.

\section{Level of Consciousness at Time of ICD Discharge}

Although the ICD markedly improves survival in patients at risk of sudden death, it would also be desirable for the ICD to terminate VT or VF before a patient loses consciousness. In this study, the majority of patients who were not treated with an antiarrhythmic drug did not have syncope in association with spontaneous ICD shocks. Not only was antiarrhythmic drug therapy not associated with a reduction in the incidence of syncope, it may have resulted in a higher incidence of syncope in association with ICD shocks. Because the mean rates of induced VT and the incidence of treatment with beta and calcium channel blockers in group A and B were similar, the higher incidence of loss of consciousness in group A is not attributable to a difference in the baseline VT characteristics or treatment with medicines (e.g., beta and calcium channel blockers) that potentially might impair compensatory hemodynamic reflexes between the two groups of patients. Although the explanation for a higher incidence of syncope in patients treated with antiarrhythmic drugs is unclear, one possibility is that the antiarrhythmic drugs impaired cardiovascular homeostatic reflexes and resulted in a lower blood pressure during VT.

\section{Limitations}

The major limitation of this study is that it was retrospective and that antiarrhythmic drug therapy was not assigned on a random basis. However, we were unable to detect any significant differences between the patients who were and were not treated with antiarrhythmic drugs in clinical variables that might influence the incidence of ICD shocks. Therefore, there was no obvious bias influencing the decision to treat a patient with an antiarrhythmic drug. A possible bias towards treating patients who had frequent episodes of VT or VF was avoided in this study by limiting the subjects to patient who had a history of only one episode of sustained VT or VF before undergoing implantation of the ICD.

The validity of the conclusions of this study depends on the premise that the ICD discharges were triggered by episodes of sustained VT/VF. Although every effort was made to screen out patients who had received inappropriate shocks from the ICD, the possibility exists that some of the shocks were inappropriate. However, even when all ICD shocks including appropriate and inappropriate are taken into account, there was still no difference in the incidence of shocks between patients who were and were not with antiarrhythmic drugs.

In view of a short follow-up period and small sample size, the statistical power of the Wilcoxon test to detect differences in freedom from ICD shocks between the two groups was only about $50 \%$. However, in patients who did experience ICD shocks, the frequency of shocks was similar in the two groups, further suggesting that such antiarrhythmic therapy did not have an influence on occurrence of ICD shocks. Nonetheless, confirmatory studies providing greater statistical power are clearly needed.

\section{Clinical Implications}

Although it is clear that the inability of a drug to suppress the induction of VT during electrophysiological testing is associated with a high risk of recurrent VT during chronic therapy with that agent, ${ }^{6,7}$ it has not been clear whether a drug that fails to suppress the induction of VT might have a partial beneficial effect by decreasing the number of presumed episodes of VT or VF during shortterm follow-up. Moreover, although empiric drug therapy for sustained VT/VF has been associated with a high risk of recurrent $\mathrm{VT} / \mathrm{VF},{ }^{8}$ it has also 
been unclear whether this type of treatment might reduce the incidence of VT. The results of this study suggest that agents either failed electrophysiological testing or empirically selected does not reduce the frequency of episodes of VT/VF during short-term follow-up. Given the potential toxicity and adverse effects of antiarrhythmic drugs, including a possible increase in the risk of syncope during VT, there does not appear to be a strong

\section{References}

1. Echt DS, Armstrong K, Schimdt P, et al. Clinical experience, complications, and survival in 70 patients with the automatic implantable cardioverter/defibrillator. Circulation 1985; 71:289296.

2. Marchlinski FE, Flores BT, Buxton AE, et al. The automatic implantable cardioverter defibrillator: Efficacy, complications, and device failures. Ann Int Med 1988; 104:481-488.

3. Myerburg RJ, Luceri RM, Thurer R, et al. Time to first shock and clinical outcome in patients receiving an automatic implantable cardioverterdefibrillator. J Am Coll Cardiol 1989; 14:508514.

4. Tchou PJ, Kadri N, Anderson J, et al. Automatic implantable cardioverter defibrillator and survival of patients with left ventricular dysfunction and ma- rationale for using antiarrhythmic drugs after implantation of an ICD in attempt to reduce the ICD shocks, at least in patients who have a history of only one episode of VT/VF. Studies with a larger sample size and longer follow-up period will be needed to confirm this impression.

Acknowledgment: We would like to thank Penny Weaver for her assistance in preparation of this manuscript.

lignant ventricular arrhythmias. Ann Int Med 1989;109:529-534.

5. Kelley PA, Cannon DS, Garan H, et al. Predictors of automatic implantable cardioverter defibrillator discharge for life-threatening ventricular arrhythmias. Am J Cardiol 1988; 62:83-87.

6. Kim SG, Seiden SW, Felder SD, et al. Is programmed stimulation of value in predicting the long-term success of antiarrythmic therapy for ventricular tachycardias? N Engl J Med 1988; 315:356-362.

7. Eldar M, Sauve MJ, Scheinman MN. Electrophysiologic testing and follow-up of patients with aborted sudden death. J Am Coll Cardiol 1987; 10:291-298.

8. Ferguson D, Saksena J, Greenberg E, et al. Management of recurrent ventricular tachycardia: Economic impact of therapeutic alternatives. Am J Cardiol 1984; 53:531-536. 
Copyright of Pacing \& Clinical Electrophysiology is the property of Blackwell Publishing Limited and its content may not be copied or emailed to multiple sites or posted to a listserv without the copyright holder's express written permission. However, users may print, download, or email articles for individual use. 